\title{
Depressão pós-parto e variáveis sociodemográficas em mães de bebês com e sem Síndrome de Down
}

\author{
Tahena Silva Ferreira ${ }^{1}$ \\ Bárbara Camila de Campos ${ }^{2}$ \\ Olga Maria Piazentin Rolim Rodrigues ${ }^{3}$ \\ ${ }^{1}$ Universidade Estadual Paulista "Júlio, de Mesquita Filho”, Bauru, SP, Brasil \\ https://orcid.org/0000-0002-6089-9498 \\ ${ }^{2}$ Universidade Estadual Paulista "Júlio, de Mesquita Filho”, Bauru, SP, Brasil \\ https://orcid.org/0000-0003-4856-1491 \\ ${ }^{3}$ Universidade Estadual Paulista "Júlio, de Mesquita Filho", Bauru, SP, Brasil \\ https://orcid.org/0000-0002-5332-256X
}

\begin{abstract}
Resumo
A depressão que se inicia no puerpério é denominada como depressão pós-parto materna (DPP-M). A Síndrome de Down (SD) é uma cromossomopatia de alta incidência que ocasiona manifestações físicas, clínicas e intelectuais específicas. Objetivou-se descrever e comparar a frequência da DPP-M e de características sociodemográficas em mães de bebês com e sem SD e identificar possíveis preditores da DPP-M entre as variáveis sociodemográficas maternas, do bebê e familiares. Participaram 60 díades. Utilizou-se um questionário sociodemográfico e a Edinburgh Postpartum Depression Scale (EPDS). Os resultados não indicaram diferença significativa nos índices de DPP-M dos grupos, sendo esses mais elevados em mães de bebês sem SD. Comparando as variáveis sociodemográficas, o número de filhos e a idade do bebê foram maiores entre mães de bebês com SD e mais mães de bebês sem SD trabalham fora. A escolaridade, o tipo de família e a idade do bebê foram preditores para DPP-M.
\end{abstract}

Palavras-chave: depressão pós-parto, mães-bebês, Síndrome de Down.

\section{Postpartum depression and sociodemographic variables in mothers of babies with and without Down's Syndrome}

\begin{abstract}
Depression that initiates at puerperium is denominated maternal depressive post-partum disorder (M-DPPD). Down Syndrome (DS) is a chromosopathy of high incidence that occasionates specific physical, clinical and intellectual manifestations. This study aimed to describe and compare the frequency of M-DPPD and sociodemographic characteristics in mothers of babies with and without DS and identify possible predictors of M-DPPD among maternal, baby and family sociodemographic variables. Participants were 60 dyads. A sociodemographic questionnaire and the Edinburgh Postpartum Depression Scale (EPDS) were used. The results did not showed a significant difference in the M-DPPD indices of the groups, being these higher in mothers of infants without SD. Comparing the sociodemographic variables, the number of children and the age of the baby were higher among mothers of babies with SD and more mothers of babies without SD working outside. Schooling, family type, and baby's age were predictors for DPP-M.
\end{abstract}

Keywords: postpartum depression, mothers-babies, Down's Syndrome. 


\title{
Depresión post-parto y variables sociodemográficas en madre de bebés con y sin Síndrome de Down
}

\begin{abstract}
Resumen
La depresión que se inicia en el puerperio es denominada depresión posparto materna (DPP-M). El síndrome de Down es una cromosomopatía de alta incidencia que ocasiona un conjunto de manifestaciones físicas, clínicas y intelectuales específicas. Se objetivó describir y comparar la frecuencia de la DPP-M y de características sociodemográficas en madres de bebés con y sin SD e identificar posibles predictores de la DPP-M entre las variables sociodemográficas maternas, del bebé y familiares. Participaron 60 diadas. Se utilizó un cuestionario sociodemográfico y la Edinburgh Postpartum Depression Scale (EPDS). Los resultados señalaron que no hubo diferencia significativa en el índice de DPP-M de los grupos, siendo este índice más elevado en madres de bebés sin SD. Comparando las variables sociodemográficas, el número de hijos y la edad del bebé fueron mayores entre madres de bebés con SD y más madres de bebés sin SD trabajan fuera. La escolaridad, el tipo de familia y la edad del bebé fueron predictores para DPP-M.
\end{abstract}

Palabras clave: depresión postparto, madre e hijo, Sindrome de Down.

O nascimento de uma criança é permeado de comemorações e alegrias. Porém, requer grandes transformações na vida da mulher, podendo gerar riscos potenciais para o desenvolvimento de transtornos psicológicos (Dois et al., 2012). Assim, a gestação e o puerpério configuram-se em fatores de risco para apresentação ou aumento de transtornos dessa ordem, especialmente, da depressão pós-parto (Lobato, Moraes, \& Reichenheim, 2011; Sit \& Wisner, 2009; American Psychological Association [APA], 2013).

A depressão que se inicia no puerpério é denominada transtorno depressivo no pós-parto ou depressão pós-parto materna (DPP-M). Esse transtorno pode se manifestar nas primeiras quatro semanas após o parto (APA, 2013), porém, estudos defendem a extensão dessa condição para seis, doze ou até vinte e quatro meses após o nascimento do bebê (Austin et al., 2014; Cohen et al., 2010; Frizzo \& Piccinini, 2005; Horowitz \& Goodman, 2004).

A DPP-M é uma das principais causas de morbidade materna, sendo a complicação médica mais comum entre mulheres durante seu período reprodutivo (Lobato, Moraes, \& Reichenheim, 2011; Campos \& Rodrigues, 2015). Dados de uma revisão sistemática indicaram que $19,8 \%$ das mulheres que vivem em países em desenvolvimento podem apresentar depressão após o parto (Fisher et al., 2012). No Brasil, um estudo realizado entre fevereiro de 2011 e outubro de 2012 com 23.894 mulheres em um período de seis horas do parto e até 18 meses após nascimento da criança, apontou uma prevalência de possíveis casos de DPP-M em 26,3\% dessa amostra (Filha, Ayers, \& Carmo Leal, 2016).

A etiologia da DPP-M envolve uma série de fatores de risco (Miller \& La Russo, 2011). As análises sugerem alguns fatores preditivos para essa condição, como: história anterior de depressão (Faisal-Cury \& Menezes, 2012; Fisher et al., 2012; Defelipe et al., 2017; Morais et al., 2015), desvantagem socioeconômica, gravidez indesejada, falta de empatia e apoio do parceiro íntimo (Fisher et al., 2012; Defelipe et al., 2017; Filha, Ayers, \& Carmo Leal, 2016), conflito conjugal (Defelipe et al., 2017; Morais et al., 2015), eventos estressantes da vida, baixa autoestima, problemas de viabilidade infantil (Defelipe et al., 2017) e o nascimento de bebês com anomalia (Silva et al., 2018).

Esses fatores de risco parecem ser estáveis em todas as culturas (Filha, Ayers, \& Carmo Leal, 2016). No Brasil, destacam-se especialmente, o baixo nível socioeconômico, a alta paridade, não morar com o parceiro, os transtornos psiquiátricos/psicológicos prévios e a gravidez não planejada como fatores de risco para a DPP-M (Silva et al., 2012, Melo Jr et al., 2012).

Dentre os impactos da DPP-M destacam-se os prejuízos na amamentação, no desenvolvimento social, emocional e cognitivo da criança e, ainda, no estabelecimento do vínculo entre mãe e filho (Moehler et al., 2006). A exposição prolongada da criança aos efeitos da depressão pós-parto afeta as fases posteriores de seu 
desenvolvimento e está associada a distúrbios emocionais na infância e na adolescência (Santos et al., 2014).

Diversos estudos que relacionaram a saúde mental materna aos indicadores de risco para o desenvolvimento infantil foram realizados em âmbito mundial. Na Grécia, Giakoumaki et al. (2009), nos Estados Unidos, Arteche et al. (2011), em Israel, Reck et al. (2012), no Reino Unido, Glover (2014), na França, Eutrope et al. (2014) e, em Taiwan, Liou, Wang e Cheng (2014). Todavia, grande parte dos estudos sobre DPP-M relacionam essa condição a bebês saudáveis, visto que bebês que apresentam algum problema de saúde ou apresentam uma malformação tendem a ficar internados após o nascimento e são automaticamente, excluídos das pesquisas (Azambuja, Cardoso, \& Da Silva, 2016). Dentre as possíveis malformações que podem acometer o bebê está a Síndrome de Down (SD).

A SD é considerada um tipo de cromossomopatia causada em 95\% dos casos pela presença de três cromossomos 21 (e não dois, como no desenvolvimento típico). Além da trissomia do 21, há a translocação, verificada em $4 \%$ dos casos, sendo considerada uma alteração cromossômica estrutural e o mosaicismo, que representa $1 \%$ dos diagnósticos e ocorre quando as células trissômicas aparecem ao lado das células normais (Soares et al., 2016). A SD apresenta alta incidência na população mundial (um para cada 800 indivíduos nascidos vivos) (Stedile, Da Silva, \& Hartmann, 2013). Esse índice se assemelha aos dados apresentados no Brasil, onde é registrado o nascimento de uma criança portadora da síndrome para cada 600 a 800 nascidos vivos (Brasil, 2012).

Essas alterações cromossômicas resultam em um conjunto de manifestações físicas, clínicas e mentais específicas, podendo afetar indivíduos de diversas raças, etnias e classes socioeconômicas, sendo ainda, considerada a causa mais frequente de deficiência intelectual (Freire, Melo, Hazin \& Lyra, 2014). A SD apresenta um fenótipo característico, representado por mãos pequenas e dedos curtos, pés com espaçamento entre o primeiro e o segundo dedo, prega palmar única, hipotonia muscular, nariz pequeno, face com perfil achatado, pregas epicânticas, manchas de Brushfield, orelhas pequenas e lóbulos praticamente inexistentes, excesso de pele na nuca, boca pequena e falta de tônus muscular, língua geográfica, dentes pequenos e com formas anormais, pele com aspecto manchado e tendência a ressecamento com o passar dos anos, cabelo fino e ralo, atraso no crescimento e, além disso, a cabeça também pode apresentar tamanhos menores do que os considerados normais. Todavia, é importante ressaltar que raramente todas as características apresentadas são evidentes na mesma criança (Koch \& Silva, 2016). Essa população também apresenta manifestações clínicas que derivam de problemas associados ao atraso no desenvolvimento, especialmente a cardiopatia congênita e problemas gastrointestinais (Chan et al., 2015). Além das características fenotípicas e clínicas, a criança com SD, de modo geral, apresenta um atraso no seu desenvolvimento global, isto é, tanto as funções neuropsicomotoras quanto as cognitivas são prejudicadas, caracterizando uma deficiência, principalmente intelectual e limitando comportamentos adaptativos expressos por meio de habilidades práticas, conceituais e sociais (Vital et al., 2015).

Em decorrência das implicações e desafios vinculados a criação de uma criança com SD, a deficiência pode configurar-se em um fator de risco e comprometer a saúde emocional materna (Majid \& Sareskanrud, 2012; Choi \& Yoo, 2015; Nes et al., 2014). Todavia, são poucos os estudos que relacionam a DPP-M e bebês com malformações, o que inclui, a SD. Uma revisão sistemática desenvolvida por Azambuja, Cardoso e da Silva (2016) apontou que não houve nenhum estudo que investigasse a DPP-M em bebês com malformação nos últimos dez anos. Esse dado evidencia a escassez de pesquisas nessa área, especialmente, nas idades mais precoces e que incluam o desenvolvimento atípico.

Fairthorne et al. (2015) compararam a incidência de diagnósticos de transtornos psiquiátricos em mães após o nascimento de um filho com deficiência. As mães e seus filhos, foram separados em cinco grupos: 1) Mães e filhos sem deficiência); 2) Mães e filhos com incapacidade intelectual leve a moderada de causa desconhecida; 3 ) 
Mães e filhos com deficiência intelectual grave de causa desconhecida; 4) Mães e filhos com Síndrome de Down; e 5) Mães e filhos com deficiência intelectual de causa conhecida excluindo síndrome de Down. Também foram avaliadas variáveis consideradas como preditoras de risco para o desenvolvimento infantil, como: desvantagem socioeconômica; idade materna menor e maior número de filhos. Participaram 277.559 mães que deram à luz a uma criança viva na Austrália Ocidental entre 1 de janeiro de 1983 e 31 de dezembro de 2005. Os resultados apontaram que mães de filhos com Síndrome de Down apresentaram taxas reduzidas em todas as categorias psiquiátricas analisadas (álcool e abuso de substâncias; transtornos esquizoides; transtornos afetivos; distúrbios neuróticos e outros distúrbios psiquiátricos), não sendo verificados resultados com significância estatística.

Tekinarslan (2013) comparou a qualidade de vida e os níveis de depressão em mães turcas de crianças e adolescentes com Síndrome de Down, paralisia cerebral e Transtorno do Espectro Autista, com idades entre três e dezoito anos. Participaram 252 mães, sendo que 93 tinham filhos autistas, 78 com Síndrome de Down e, 81, com paralisia cerebral. Para avaliar a qualidade de vida foi utilizado o WHOQOL-BREF-TR da Organização Mundial de Saúde e para investigar a depressão utilizou-se o Inventário de Depressão de Beck (BDI). Os resultados apontaram que não houve uma diferença significativa para depressão materna entre os grupos. Considerando as variáveis sociodemográficas, foram observadas diferenças significativas, sendo os menores escores médios para depressão observados em mães mais jovens (até 25 anos), que possuíam graduação e/ou pós-graduação, renda alta e união estável. Para comparar a saúde mental de mães de crianças com SD com seus pais e com mães de crianças sem a síndrome, Hedov, Annéren e Wikblad (2000) desenvolveram um estudo utilizando o Swedish SF36 Health Survey. Os resultados apontaram para menos indicadores de saúde mental das mães de crianças com $\mathrm{SD}$ do que os outros grupos comparativos.
Um estudo foi desenvolvido por Bourke et al. (2008) para investigar se o estado de saúde, a comunicação, o comportamento e o nível de funcionamento de crianças até adultos jovens com Síndrome de Down estavam associados a saúde mental materna. Participaram do estudo 250 mães de filhos com SD entre zero e 25 anos que responderam a um questionário com perguntas relacionadas à saúde, ao funcionamento e às necessidades do filho e ao 12-Item Short-Form Health Survey (SF-12) que aborda a saúde e o bem-estar da família. Esse instrumento avalia oito dimensões de influência sobre a qualidade de vida (função física, aspecto físico, dor, saúde geral, vitalidade, função social, aspecto emocional e saúde mental), considerando a percepção dos indivíduos nas quatro últimas semanas. Os dados dessa escala são mensurados em duas categorias: o físico (Physical Component Summary ou PCS) e o mental (Mental Component Summary ou MCS). A pontuação de ambas as escalas varia entre zero e 100, sendo os maiores escores associados a melhores níveis de qualidade de vida. Análises de regressão linear foram feitas com as duas categorias (PCS e MCS), tendo sido selecionadas as variáveis do estado de saúde, comportamento, comunicação e função da criança que os pesquisadores consideraram que teriam maior efeito sobre a saúde materna, entre eles: a existência de problemas no coração ou intestino, o número de problemas de saúde, o número de episódios da doença no último ano, o nível de comunicação e compreensão e o nível de independência das atividades de vida diária. As condições sociodemográficas tanto da mãe quanto do filho também foram consideradas. Os resultados apontaram que o índice do PCS materno foi de 50,2 pontos, significativamente maior que a média feminina australiana $(\mathrm{p}=0,015)$. A análise do MCS indicou um escore médio materno de 45,2 pontos, considerado significativamente menor que a média feminina australiana $(51,42)(\mathrm{p}<0,0001)$. A saúde mental da mãe melhorou com a idade materna, todavia, após o ajuste do efeito da idade da mãe na saúde mental, a idade da criança não foi significativa na saúde mental materna. Os piores índices de saúde mental materna 
foram associados às mães de crianças com Síndrome de Down com problemas auditivos, musculares e ósseos que ocorreram durante a pesquisa. Ainda, foram observados escores de saúde mental significativamente mais baixos em mães de crianças com SD que apresentaram problemas de personalidade disfuncional, emocionais ou de comportamento $(p<0,001)$. Maiores escores de saúde mental relacionados à capacidade funcional das crianças com SD foram observados em mães cujos filhos eram mais velhos, precisando, apenas de supervisão (não de ajuda) para execução das tarefas. Desta forma, o presente estudo apontou que a saúde mental das mães foi fortemente influenciada pelo comportamento infantil e pelas demandas de cuidado. Enquanto os preditores mais importantes da saúde materna, especialmente a mental, foram as dificuldades comportamentais da criança, o seu nível de funcionamento para desenvolver as atividades de vida diária, o seu progresso no convívio social e, em menor grau, a sua saúde física.

Embora a depressão pós-parto seja considerada um dos problemas de saúde mais comuns entre as mulheres (Lobato, Moraes, \& Reichenheim, 2011), ainda é possível perceber uma importante lacuna referente aos estudos que investiguem os efeitos dessa condição em relação às mães de bebês com desenvolvimento atípico, sobretudo, com Síndrome de Down. Tais estudos podem contribuir para identificação da prevalência e de preditores da DPP-M, o que permitiria o planejamento de intervenções preventivas e precoces de saúde, aumentando a qualidade de vida das mães e minimizando os prejuízos no desenvolvimento infantil.

Desta forma, o objetivo do presente estudo foi descrever e comparar a frequência da depressão pós-parto e as características sociodemográficas em mães de bebês com e sem Síndrome de Down, bem como identificar possíveis preditores da depressão pós-parto, entre as variáveis sociodemográficas maternas, do bebê e familiares para a amostra toda.

\section{Método}

O presente estudo é descritivo, envolvendo comparação entre grupos e análise de correlação.

\section{Participantes}

Participaram desse estudo 60 mães que faziam parte do projeto "Variáveis do bebê e maternas: correlação com interação mãe-bebê e desenvolvimento infantil" aprovado pelo Comitê de Ética (Processo n. . 11187/46/01/2012), da Faculdade de Ciências, UNESP, Bauru (n. . 4205/46/01/11). Delas, 30 mães de bebês com Síndrome de Down foram identificadas em instituições de Londrina/PR e Curitiba/ $\mathrm{PR}$, que atendiam crianças com SD e compuseram o G1. O critério de inclusão foi que os bebês não tivessem outras comorbidades que não estivessem associadas especificamente à síndrome, constituindo uma amostra por conveniência. As demais, mães de crianças sem a síndrome ou outros problemas de desenvolvimento ou saúde, eram participantes de um projeto de extensão que monitora o desenvolvimento de bebês e na mesma época foram convidadas a responder a EPDS, portanto, também uma amostra de conveniência. Quanto às variáveis sociodemográficas, $63 \%$ das mães do G1 e $90 \%$ das mães do G2 tinham 33 anos ou mais de idade. Quanto ao estado civil 93\% das mães dos dois grupos relataram ter uma união estável e, também, $80 \%$ das mães do G1 e $83 \%$ das mães do G2 moravam somente com o marido e os filhos. Considerando a escolaridade, $83 \%$ das mães dos dois grupos tinham pelo menos o Ensino Médio completo. Das mães do G1, 40\% e, do G2, 83\% trabalham fora do lar. Quanto ao tipo de parto, 60\% das mães do G1 e 57\% das mães do G2, fizeram cesárea. Das características dos bebês, $67 \%$ dos bebês do G1 e $50 \%$ do G2 eram meninos. Dos bebês do G1 53\% eram primeiro ou segundo filho e, $86 \%$ do G2 também o eram.

\section{Local}

A aplicação dos instrumentos ocorreu em salas de atendimento individual nas instituições que atendem pessoas 
com SD e no Centro de Psicologia Aplicada, garantindo privacidade e condições favoráveis para a pesquisa.

\section{Materiais}

O Instrumento para Coleta de Informações Sociodemográficas (ICIS), elaborado para este estudo, é composto por questões sobre dados pessoais como: idade, escolaridade, número de filhos, estado civil, trabalho remunerado, entre outros.

Para a investigação da depressão pós-parto, foi utilizada a Edinburgh Postpartum Depression Scale (EPDS), de Cox, Holden e Sagovsky (1987), validada e adaptada para o Brasil por Santos, Martins e Pasquali (1999). Trata-se de um instrumento de autopreenchimento, composto por 10 afirmações. Para cada uma delas são propostas quatro frases, devendo ser escolhida aquela que mais se assemelha aos estados emocionais maternos percebidos na última semana. Cada uma das quatro alternativas propostas é associada a uma pontuação, entre zero e três. O escore final do instrumento é calculado com base na somatória da pontuação obtida em cada uma das 10 afirmações propostas, que pode variar entre zero e 30 pontos. Seguindo as diretrizes de Santos, Martins e Pasquali (1999), para a população brasileira, um escore de 10 pontos já é indicativo de depressão pós-parto leve, a partir de 12 pontos há uma indicação de depressão moderada a severa.

\section{Procedimento para a coleta dos dados}

Após contato com as instituições que atendiam crianças com SD foram identificadas aquelas que preenchiam o critério de idade (de três a sete meses). Foi feito contato via telefone pela pesquisadora convidando a mãe para participar do projeto. Em caso de aceite foi agendado dia e hora para que respondesse aos instrumentos. No projeto de extensão, as mães que participavam e que tinham bebês na mesma faixa etária também eram convidadas. Aceitando o convite, a participante respondia aos instrumentos. Em todos os casos, em uma sala de atendimento individual, após a apresentação do proje- to e esclarecimento de dúvidas, as mães assinavam o Termo de Consentimento Livre e Esclarecido (TCLE) e respondiam aos materiais usados neste estudo. O tempo de aplicação variou de 20 a 30 minutos. Todas as mães que apresentaram escores iguais ou superiores a 12 pontos nessa escala, foram encaminhadas para serviços de Psicologia das Unidades de Saúde a que pertenciam.

\section{Procedimento para a análise dos dados}

Os valores atribuídos pelas participantes em cada afirmação da EPDS foram somados. Foi adotado o ponto de corte igual ou superior a 12 pontos e, para este trabalho, nas análises qualitativas, os dados provenientes da escala foram categorizados de acordo com o ponto de corte em clínico e não clínico. Os dados sociodemográficos coletados foram tabulados, categorizados e submetidos a análises descritivas de suas frequências. Para verificação da normalidade foi utilizado o teste de Shapiro-Wilk. Para verificar a diferença dentre grupos considerando o escore na EPDS foi utilizado o Teste $t$ para dados não paramétricos. Para verificar a correlação entre a pontuação obtida na EPDS e as variáveis sociodemográficas maternas quantitativas foi utilizado o teste de Spearman. E, ainda, para a verificação de uma possível associação entre a EPDS e as variáveis sociodemográficas maternas foi utilizada a Regressão Linear Múltipla. Todos os testes foram performados com base em um nível de significância de 5\%.

\section{Resultados}

As frequências dos níveis de depressão baseados na EPDS para os dois grupos são apresentadas na Tabela 1. Observou-se diferença entre as frequências apresentadas pelos grupos, todavia, ela não foi estatisticamente significativa $(p=0,1082)$. Observa-se que das trinta mães de bebês com SD, apenas cinco apresentaram risco para depressão pós-parto. Dentre as trinta mães de bebês sem SD, o risco (12 ou mais pontos) pode ser verificado em doze delas. Para o G1, díades com 
bebês com Síndrome de Down, a média de pontos na a média de pontos obtidas na EPDS foi de oito pontos escala foi de 6,47 pontos (Min.=0; $\operatorname{Max}=20 ; \mathrm{DP}=4,49) . \quad(\operatorname{Min} .=1 ; \operatorname{Max}=18 ; \mathrm{DP}=4,84)$.

Enquanto, no G2, díades com bebês sem a síndrome,

\section{Tabela 1}

Estatísticas descritivas (média e desvio padrão) e significância entre o G1 e G2, considerando os escores na EPDS

\begin{tabular}{|c|c|c|c|c|c|c|}
\hline & \multicolumn{2}{|c|}{ G1 } & \multicolumn{2}{|c|}{ G2 } & \multirow[t]{2}{*}{ t } & \multirow[t]{2}{*}{$\mathbf{p}$} \\
\hline & $n=30(100 \%)$ & $\mathbf{M}(\mathbf{D P})$ & $\mathrm{n}=30(100 \%)$ & $\mathbf{M}(\mathbf{D P})$ & & \\
\hline Clínico & $5(16,6)$ & \multirow{2}{*}{$6,46(4,57)$} & $12(40)$ & \multirow{2}{*}{$8(4,93)$} & \multirow{2}{*}{$-1,249$} & \multirow{2}{*}{0,216} \\
\hline Não clínico & $25(83,4)$ & & $18(60)$ & & & \\
\hline
\end{tabular}

T: Teste-T; p: significância (0,005); M=Média; DP=Desvio Padrão

A Tabela 2 apresenta a comparação entre as variáveis contínuas sociodemográficas maternas e dos bebês dos dois grupos. As mães dos bebês sem a síndrome são mais velhas e as mães de bebês com Síndrome de Down têm maior escolaridade, mais filhos e o bebê era mais velho por ocasião da coleta. Todavia, observou-se diferenças significativas entre os grupos quanto a número de filhos $(\mathrm{p}=0,013)$ e idade do bebê $(p=0,000)$.

\section{Tabela 2}

Estatísticas descritivas (média e desvio padrão) e significância entre o G1 e G2, considerando as variáveis contínuas sociodemográficas

\begin{tabular}{|l|c|c|c|c|c|c|}
\hline & \multicolumn{2}{|c|}{ G1 } & \multicolumn{2}{c|}{ G2 } & & \\
\hline $\begin{array}{l}\text { Variáveis } \\
\text { (N=60) }\end{array}$ & Média & DP & Média & DP & t & p \\
\hline $\begin{array}{l}\text { Idade } \\
\text { materna }\end{array}$ & 34,83 & 5,90 & 37,40 & 3,12 & 1,661 & 0,105 \\
\hline $\begin{array}{l}\text { Quantidade } \\
\text { de anos de } \\
\text { estudo }\end{array}$ & 12,34 & 2,96 & 9,92 & 3,29 & $-0,859$ & 0,392 \\
\hline $\begin{array}{l}\text { Número de } \\
\text { filhos }\end{array}$ & 2,40 & 0,77 & 1,70 & 0,91 & 2,551 & 0,013 \\
\hline $\begin{array}{l}\text { Idade do } \\
\text { bebê }\end{array}$ & 5,70 & 1,87 & 4,36 & 0,37 & 4,868 & 0,000 \\
\hline
\end{tabular}

T: Teste-T; p: significância (0,005); DP=Desvio Padrão
Considerando as variáveis categóricas sociodemográficas (Tabela 3) observou-se diferença significativa quanto ao trabalho formal $(\mathrm{p}=0,001)$. Mais do que o dobro das mães do G2 trabalha comparando com as mães do G1. As demais variáveis analisadas (estado civil, tipo de parto e sexo do bebê) foram equivalentes para os dois grupos.

\section{Tabela 3.}

Estatísticas descritivas e significância entre G1 e G2, considerando as variáveis categóricas sociodemográficas

\begin{tabular}{|c|c|c|c|c|c|}
\hline & & G1 & G2 & $\mathbf{X}^{2}$ & $\mathbf{P}$ \\
\hline $\begin{array}{l}\text { Variáveis } \\
(\mathrm{N}=60)\end{array}$ & & N (\%) & N (\%) & & \\
\hline Estado civil & Solteira & $2(7)$ & $3(10)$ & \multirow[b]{2}{*}{0,218} & \multirow[b]{2}{*}{0,640} \\
\hline & $\begin{array}{l}\text { União } \\
\text { Estável }\end{array}$ & $\begin{array}{c}28 \\
(93)\end{array}$ & $\begin{array}{c}27 \\
(90)\end{array}$ & & \\
\hline \multirow[t]{2}{*}{$\begin{array}{l}\text { Trabalhadora } \\
\text { formal }\end{array}$} & Sim & $\begin{array}{c}12 \\
(40)\end{array}$ & $\begin{array}{c}25 \\
(83)\end{array}$ & \multirow{2}{*}{11,915} & \multirow{2}{*}{0,001} \\
\hline & Não & $\begin{array}{c}18 \\
(60)\end{array}$ & $5(17)$ & & \\
\hline \multirow[t]{2}{*}{ Tipo de parto } & Natural & $\begin{array}{c}12 \\
(40)\end{array}$ & $\begin{array}{c}13 \\
(43)\end{array}$ & \multirow{2}{*}{0,069} & \multirow{2}{*}{0,793} \\
\hline & Cesárea & $\begin{array}{c}18 \\
(60)\end{array}$ & $\begin{array}{c}17 \\
(57)\end{array}$ & & \\
\hline
\end{tabular}




\begin{tabular}{|l|l|c|c|l|l|}
\hline Sexo do bebê & Feminino & $\begin{array}{c}20 \\
(33)\end{array}$ & $\begin{array}{c}15 \\
(50)\end{array}$ & \multirow{2}{*}{1,714} & \multirow{2}{*}{0,190} \\
\hline & Masculino & $\begin{array}{c}10 \\
(67)\end{array}$ & $\begin{array}{c}15 \\
(50)\end{array}$ & & \\
\hline
\end{tabular}

$\mathbf{X}^{\mathbf{2}}=$ qui-quadrado; $\mathrm{p}=$ significância $(0,005)$

Considerando-se que para os grupos não foram observadas diferenças significativas em relação ao índice de depressão pós-parto em comparação à condição da presença ou ausência da Síndrome de Down nos bebês, todas as características sociodemográficas da amostra total foram colocadas em modelos de regressão linear múltipla com o objetivo de identificar possíveis variáveis preditoras da depressão pós-parto. A análise resultou em um modelo final que apresentou apenas as variáveis significativas (Escolaridade, Tipo de família e Idade do bebê) e que explicaram $25 \%$ da ocorrência de depressão pós-parto. Segundo a Tabela 4, a menor escolaridade materna tem relevância de 39,10\% de predição, ser solteira explica $36,40 \%$ e bebês mais novos indicam um grau de predição de $24,50 \%$.

\section{Tabela 4}

Regressão linear múltipla das variáveis maternas, do bebê e familiares preditoras de índice clínico na EPDS nos dois grupos

\begin{tabular}{|c|c|c|c|c|c|}
\hline $\begin{array}{c}\text { Variáveis } \\
\text { dependentes }\end{array}$ & $\mathbf{R}$ & $\mathbf{R 2}$ & Beta & $\mathbf{t}$ & $\mathbf{p}$ \\
\hline $\begin{array}{c}\text { Variáveis } \\
\text { independentes }\end{array}$ & Ajusted & & & \\
\hline Escolaridade & 0,391 & 0,166 & $-3,341$ & $-3,134$ & 0,004 \\
\hline Tipo de família & 0,364 & 0,187 & 8,61 & 0,061 & 0,006 \\
\hline Idade do bebê & 0,245 & 0,325 & $-2,901$ & $-1,147$ & 0,022 \\
\hline
\end{tabular}

\section{Discussão}

Desta forma, o objetivo do presente estudo foi descrever e comparar a frequência da depressão pós-parto e as características sociodemográficas em mães de bebês com e sem Síndrome de Down, bem como identificar possíveis preditores da depressão pós-parto, entre as variáveis sociodemográficas maternas, do bebê e familiares para a amostra toda.

Esse estudo comparou os índices de DPP-M em mães de bebês com e sem Síndrome de Down e não foram encontradas diferenças significativas entre os dois grupos. Considerando as médias, as mães de bebês sem a síndrome apresentaram índices de risco maiores do que as mães de bebês com ela. Estudos conduzidos por Hedov, Annéren e Wikblad (2000) encontraram resultados semelhantes. Comparando a saúde mental de mães de crianças com SD com mães de crianças com outras formas de deficiência, Hodapp et al. (2001), Van Riper et al. (1992) encontraram dados que sugerem que a saúde mental das primeiras é menos prejudicada do que a saúde mental de mães de crianças com outras formas de deficiência intelectual.

Entre as hipóteses para os achados desse estudo, uma pode estar relacionada a possibilidade do diagnóstico da SD ser realizado precocemente, o que implica na necessidade de reorganização da família para atender às necessidades da criança. Uma outra hipótese é a disponibilidade de acolhimento psicológico relativamente frequente nas instituições eletivas que atendem crianças com malformação específica, como é o caso das instituições de origem das mães. Hartmann, Mendonza-Sassi e Cesar (2017) sugerem que o apoio profissional é um fator de proteção para a depressão pós-parto. Um dos principais fatores de proteção para a depressão pós-parto é a percepção de apoio social e afetivo. Assim, podemos considerar que, por ocasião da notícia da condição de seu bebê, embora estivessem em uma situação inicial de vulnerabilidade, a reorganização e a assistência prestada a essas mulheres podem ter sido um importante fator de proteção, que justificaria a maior resiliência deste grupo.

Comparando as características sociodemográficas dos dois grupos observou-se que as mães do G1 têm mais filhos e os bebês eram mais velhos por ocasião da coleta. A idade mais avançada dos bebês do G1 se deve às muitas remarcações dos encontros que chegaram a 
demorar até dois meses. No entanto, para garantir o número esperado de participantes optou-se por esperar, mesmo que os bebês fossem um pouco mais velhos. As mães do G2 trabalham mais fora do que as mães do G1. Considerando que elas têm nível de escolaridade semelhante, uma hipótese é que pode ficar difícil associar todos os atendimentos que o bebê necessita com os horários de trabalho, o que justificaria não estarem envolvidas em trabalho formal nessa idade do bebê.

A análise de regressão foi feita com a amostra toda considerando a presença/ausência da SD como uma variável a ser associada também com a variável de desfecho, a depressão. Todavia, confirmando a não diferença entre os grupos, a presença da SD não foi significativa para a depressão pós-parto. Foram variáveis significativas a escolaridade, o tipo de família e a idade dos bebês.

O nível educacional da mãe foi relacionado como um fator de risco para depressão pós-parto, fato que vai ao encontro das evidências na literatura (Alvarenga et al., 2012; Morais et al., 2015). A baixa escolaridade e o nível socioeconômico, no Brasil, relacionam-se com as condições de acesso a tratamentos médicos adequados durante a gravidez. A qualidade do pré-natal pode influenciar na prevenção e tratamento precoce de problemas de saúde decorrentes do período. Essa variável materna também teve correlação com o índice na EPDS e reflete as condições da rede de suporte social oferecida as gestantes (Coutinho et al., 2014; Morais et al., 2015).

Ser de família nuclear mostrou ser um fator de proteção para a depressão. Outras configurações familiares mostraram-se de risco. Haltmann et al. (2017) encontrou que mães solteiras ou que vivem em famílias numerosas têm maior tendência a depressão. Todavia, os autores alertam que se há conflitos com o cônjuge aumenta o risco para depressão. No presente estudo esse dado não foi coletado.

Ter um bebê mais novo apresentou-se, neste estudo, como fator de risco para depressão. Todavia, não foram encontrados estudos que confirmem ou refutem esse dado. Frizzo et al. (2010) e Souza, Souza e Rodrigues (2013) ressaltam que os meses iniciais da maternidade podem gerar insegurança considerando os novos papéis (ser uma boa mãe), e os antigos (ser uma boa esposa) acrescido das mudanças físicas, a retomada das atividades diárias e, muitas vezes o retorno ao trabalho. Essa insegurança, se não equacionada, pode resultar em transtornos psicológicos mais graves.

\section{Considerações finais}

Em resumo, os achados do presente estudo, quanto aos índices de DPP-M em mães de bebês com e sem SD e as possíveis correlações com as variáveis sociodemográficas indicaram uma frequência menor de risco para a DPP-M no grupo de mães de bebês com SD. Esses dados podem ser justificados pelo diagnóstico precoce e os avanços nos estudos relacionados à síndrome, permitindo a família ser direcionada e assistida durante todo o período gestacional e após o nascimento da criança, favorecendo um ajustamento das expectativas parentais diante do desenvolvimento dos seus filhos.

Desse modo, o presente estudo não confirmou o valor preditivo da deficiência sobre os índices de depressão pós-parto presentes em alguns dos estudos encontrados (Silva et al., 2018). Os achados do presente estudo chamam a atenção para a necessidade de se investigar as relações entre a depressão pós-parto e a saúde mental materna considerando a importância de intervenções precoces enquanto suporte materno e familiar, independente da presença de alguma malformação do bebê, como a Síndrome de Down.

Dentre as contribuições do presente estudo, estão a comparação e a correlação entre duas variáveis com grande incidência (DPP-M e SD) em âmbito nacional e mundial, indicando preditores de índices clínicos para a depressão pós-parto para mães de bebês com essa condição. Além disso, reforça a importância do apoio e do suporte materno nos períodos pré, peri e pós-natal. Deve-se destacar, também, que a medida da provável depressão com a Escala de Depressão Pós- 
Parto de Edimburgo tem sido amplamente utilizada e validada tanto no Brasil quanto em outros países.

As limitações do estudo são que os resultados apresentados são específicos da presente amostra. As mães de bebês com SD eram assistidas e os seus bebês frequentavam intervenções precoces em instituições com especialidades multiprofissionais da saúde. Por fim, é reconhecida a necessidade de estudos futuros com a ampliação amostral. Estudos futuros de caráter longitudinal também são sugeridos para o refinamento de possíveis fatores de proteção para intervenções precoces e pontuais diante das necessidades dessa população. Também se sugere, se possível, trabalhar com amostras aleatórias e randomizadas, o que garantiria resultados mais confiáveis do que as amostras de conveniência. Todavia, nem sempre isso é possível em populações com perfis específicos como as mães de bebês com SD. Uma outra limitação foi o uso de uma única medida para aferir a depressão pós-parto, principalmente por se tratar de um instrumento para rastreamento da depressão. Sugere-se lançar mão, em estudos futuros, de outras medidas confirmatórias.

\section{Referências}

Alvarenga, P., Dazzani, M. V. M., Alfaya, C. A. S., Lordelo, E. R., \& Piccinini, C. A. (2012). Relações entre a saúde mental da gestante e o apego materno-fetal. Estudos de Psicologia, 17(3), 477-484. doi.org/10.1590/ S1413-294X2012000300017

American Psychiatric Association (2013). Diagnostic and Statistical Manual of Mental Disorders. (5th. ed.). Arlington, VA: American Psychiatric Publishing.

Arteche, A., Joormann, J., Harvey, A., Craske, M., Gotlib, I. H., Lehtonen, A., ... Stein, A. (2011). The effects of postnatal maternal depression and anxiety on the processing of infant faces. Journal of affective disorders, 133(1), 197-203. doi.org/10.1016/j.jad.2011.04.015

Austin, M. P., \& Marcé Society Position Statement Advisory Committee. (2014). Marcé International Society position statement on psychosocial assessment and depression screening in perinatal women. Best
Practice \& Research Clinical Obstetrics \& Gynaecology, 28(1), 179-187. doi.org/10.1016/j.bpobgyn.2013.08.016

Azambuja, C. V., Cardoso, A. S., \& da Silva, R. W. S. (2016). Depressão pós-parto materna e bebês com malformações: revisão sistemática. Aletheia, 49(2).

Bourke, J., Ricciardo, B., Bebbington, A., Aiberti, K., Jacoby, P., Dyke, P., ... Leonard, H. (2008). Physical and mental health in mothers of children with Down syndrome. The Journal of pediatrics, 153(3), 320-326. doi.org/10.1016/j.jpeds.2008.02.047

Brasil. Ministério da Saúde (MS). Conselho Nacional de Saúde, \& Brasil. Ministério da Saúde (MS). Conselho Nacional de Saúde. (2013). Resolução no 466, de 12 de dezembro de 2012. Diário Oficial da União. Disponível em: http://conselho.saude.gov.br/resolucoes/2012/Reso466. pdf. Acesso em: 10 de ago. 2016. https://doi.org/10.1590/ $\underline{0102-311 \times 00086214}$

Brasil. Ministério da Saúde. (2012). Diretrizes de atenção à pessoa com Síndrome de Down. Departamento de Ações Programáticas Estratégicas. Brasília: Ministério da Saúde, DF. Disponível em: <https://rblh.fiocruz. br/sites/rblh.fiocruz.br/.../diretrizes_cuidados_sindrome_down.pdf $>$. Acesso em: 13 de nov. 2017. https://doi. org/10.11606/d.5.2011.tde-22092011-132733

Campos, B. C., \& Rodrigues, O. M. P. R. (2015). Depressão pós-parto materna: crenças, práticas de cuidado e estimulação de bebês no primeiro ano de vida. Psico, 46(4), 483-492. doi.org/10.15448/19808623.2015.4.20802

Chan, K. G., Lim, K. A., \& Ling, H. K. (2015). Care demands on mothers caring for a child with $\mathrm{D}$ own syndrome: M alaysian (S arawak) mothers' perspectives. International journal of nursing practice, 21(5), 522-531. doi.org/10.1111/ijn.12275

Choi, E. K., \& Yoo, I. Y. (2015). Resilience in families of children with D own syndrome in Korea. International Journal of Nursing Practice, 21(5), 532541. doi.org/10.1111/ijn.12321

Cohen, L. S., Wang, B., Nonacs, R., Viguera, A. C., Lemon, E. L., \& Freeman, M. P. (2010). Treatment of mood disorders during pregnancy and postpartum. Psychiatric Clinics of North America, 33(2), 273-293. doi: 10.1016/j.psc.2010.02.001 
Coutinho, L. M. S., Matijasevich, A., Scazufca, M., \& Menezes, P. R. (2014). Prevalência de transtornos mentais comuns e contexto social: análise multinível do São Paulo Ageing \& Health Study (SPAH). Cadernos de Saúde Pública, 30, 1875-1883. doi.org/10.1590/ 0102-311X00175313

Cox, J. L., Holden, J. M., \& Sagovsky, R. (1987). Detection of postnatal depression: development of the 10-item Edinburgh Postnatal Depression Scale. The British journal of psychiatry, 150(6), 782-786. doi. org/10.1192/bjp.150.6.782

Defelipe, R. P.,Resende, B. D., David, V. F., \& Bussab, V. S. R. (2017). Postpartum depression in high-risk Brazilian women: psychosocial predictors and effects on maternal vocalization. Early Child Development and Care, 1-14. https://doi.org/10.1080/03004430.2017.1389918

Dois, A., Uribe, C., Villarroel, L., \& Contreras, A. (2012). Factores de riesgo asociados a síntomas depresivos post parto en mujeres de bajo riesgo obstétrico atendidas en el sistema público. Revista médica de Chile, 140(6), 719-725. doi.org/10.4067/S0034-98872012000600004

Eutrope, J., Thierry, A., Lempp, F., Aupetit, L., Saad, S., Dodane, C., ... Rolland, A. C. (2014). Emotional reactions of mothers facing premature births: study of 100 mother-infant dyads 32 gestational weeks. PloS one, 9(8). doi.org/10.1371/journal.pone.0104093

Fairthorne, J., Jacoby, P., Bourke, J., de Klerk, N., \& Leonard, H. (2015). Onset of maternal psychiatric disorders after the birth of a child with intellectual disability: a retrospective cohort study. Journal of psychiatric research, 61, 223-230. doi.org/10.1016/j. jpsychires.2014.11.011

Faisal-Cury, A., \& Menezes, P. R. (2012). Antenatal depression strongly predicts postnatal depression in primary health care. Revista Brasileira de Psiquiatria, 34(4), 446-450. doi.org/10.1016/j.rbp.2012.01.003

Filha, M. M. T., Ayers, S., da Gama, S. G. N., \& do Carmo Leal, M. (2016). Factors associated with postpartum depressive symptomatology in Brazil: the birth in Brazil National Research Study, 2011/2012. Journal of affective disorders, 194, 159-167. doi.org/10.1016/j. jad.2016.01.020

Fisher, J., Mello, M. C. D., Patel, V., Rahman, A., Tran, T., Holton, S., \& Holmes, W. (2012). Prevalence and determinants of common perinatal mental di- sorders in women in low-and lower-middle-income countries: a systematic review. Bulletin of the World Health Organization, 90, 139-149. doi.org/10.2471/ BLT.11.091850 PMID:22423165

Freire, R. C. L., Melo, S. F., Hazin, I., \& Lyra, M. C.D.P. (2014). Aspectos neurodesenvolvimentais e relacionais do bebê com Síndrome de Down. Avances en Psicología Latinoamericana, 32(2), 247-259. doi.org/10.12804/ apl32.2.2014.05

Frizzo, G. B., \& Piccinini, C. A. (2005). Interação mãe-bebê em contexto de depressão materna: aspectos teóricos e empíricos. Psicologia em Estudo, 10(1), 47-55. doi: 10.1590/S1413-73722005000100007

Frizzo, G.B. et al. (2010) Depressão pós parto: evidência a partir de dois casos clínicos. Psicologia: Reflexão e Crítica, 23(1), 46-55. doi.org/10.1590/S010279722010000100007.

Giakoumaki, O., Vasilaki, K., Lili, L., Skouroliakou, M., \& Liosis, G. (2009). The role of maternal anxiety in the early postpartum period: screening for anxiety and depressive symptomatology in Greece. Journal of Psychosomatic Obstetrics \& Gynecology, 30(1), 21-28. doi.org/10.1080/01674820802604839

Glover, V. (2014). Maternal depression, anxiety and stress during pregnancy and child outcome; what needs to be done. Best practice \& research Clinical obstetrics \& gynaecology, 28(1), 25-35. doi.org/10.1016/j. bpobgyn.2013.08.017.

Hartmann, J.M.; Mendonza-Sassi, R.A.; Cesar, J.A. (2017) Depressão entre puérperas: prevalência e fatores associados Cadernos de Saúde Pública; 33(9), 1-10. doi: 10.1590/0102-311X00094016.

Hedov, G., Annéren, G., \& Wikblad, K. (2000) Selfperceived health in Swedish parents of children with Down's syndrome, Quality of Life Research, 9(4), 415-422. doi.org/10.1023/A:1008910527481.

Hodapp, R. M., Ly, T. M., Fidler, D. J., \& Ricci, L. A. (2001). Less stress, more rewarding: Parenting children with Down syndrome. Parenting: Science and practice, 1(4), 317-337. doi.org/10.1207/S15327922PAR0104_3

Horowitz, J. A., \& Goodman, J. (2004). A longitudinal study of maternal postpartum depression symptoms. Research and Theory for Nursing Practice, 18(2-3), 149-163. doi: 10.1891/rtnp.18.2.149.61285 
Koch, M., \& da Silva, D. R. Q. (2016). Políticas educacionais inclusivas e a síndrome de Down: Diferentes interações no contexto educacional inclusivo. Diálogo, (31), 89-103. doi.org/10.18316/2238-9024.16.26

Liou, S. R., Wang, P., \& Cheng, C. Y. (2014). Longitudinal study of perinatal maternal stress, depressive symptoms and anxiety. Midwifery, 30(6), 795-801. doi.org/10.1016/j. midw.2013.11.007

Lobato, G., Moraes, C. L., \& Reichenheim, M. E. (2011). Magnitude da depressão pós-parto no Brasil: uma revisão sistemática. Revista Brasileira de Saúde Materno Infantil, 11(4), 369-379. doi.org/10.1590/S151938292011000400003

Majid, M. A., \& Sareskanrud, M. K. (2012). A comparative study of quality of life between parents of children with Down syndrome and parents of normal children. Technical Journal of Engineering and Applied Sciences 2(3), 74-78.

Melo Jr, E. F., Cecatti, J. G., Pacagnella, R. C., Leite, D. F., Vulcani, D. E., \& Makuch, M. Y. (2012). The prevalence of perinatal depression and its associated factors in two different settings in Brazil. Journal of affective disorders, 136(3), 1204-1208. doi.org/10.1016/j. jad.2011.11.023

Miller, L. J., \& La Russo, E. M. (2011). Preventing postpartum depression. Psychiatric Clinics of North America, 34(1), 53-65. doi.org/10.1016/j.psc.2010.11.010

Moehler, E., Brunner, R., Wiebel, A., Reck, C., \& Resch, F. (2006). Maternal depressive symptoms in the postnatal period are associated with long-term impairment of mother-child bonding. Archives of women's mental health, 9(5), 273-278. doi.org/10.1007/s00737-006-0149-5

Morais, M. L. S., Fonseca, L. A. M., David, V. F., Viegas, L. M., \& Otta, E. (2015). Fatores psicossociais e sociodemográficos associados à depressão pós-parto: Um estudo em hospitais público e privado da cidade de São Paulo, Brasil. Estudos de Psicologia, 20(1), 40-49. doi.org/10.5935/1678-4669.20150006

Nes, R. B., Røysamb, E., Hauge, L. J., Kornstad, T., Landolt, M. A., Irgens, L. M., ... Vollrath, M. E. (2014). Adaptation to the birth of a child with a congenital anomaly: A prospective longitudinal study of maternal well-being and psychological distress. Developmental psychology, 50(6), 1827-1839. doi.org/10.1037/a0035996
Reck, C., Noe, D., Gerstenlauer, J., \& Stehle, E. (2012). Effects of postpartum anxiety disorders and depression on maternal self-confidence. Infant Behavior and Development, 35(2), 264-272. doi.org/10.1016/j. infbeh.2011.12.005

Santos, I. S., Matijasevich, A., Barros, A. J., \& Barros, F. C. (2014). Antenatal and postnatal maternal mood symptoms and psychiatric disorders in pre-school children from the 2004 Pelotas Birth Cohort. Journal of affective disorders, 164, 112-117. doi.org/10.1016/j.jad.2014.04.033

Santos, M. F., Martins, F. C., \& Pasquali, L. (1999). Escala de auto-avaliação de depressão pós-parto: estudo no Brasil. Revista de Psiquiatria Clínica, 26(2), 90-95.

Silva, R., Jansen, K., Souza, L., Quevedo, L., Barbosa, L., Moraes, I., ... Pinheiro, R. (2012). Sociodemographic risk factors of perinatal depression: a cohort study in the public health care system. Revista Brasileira de Psiquiatria, 34(2), 143-148. https://doi.org/10.1016/ s1516-4446(12)70031-4

Silva, H. C. D., Silva, M. D. R., Frizzo, G. B., \& Donelli, T. M. S. (2018). Sintomas Psicofuncionais e Depressão Materna: Um Estudo Qualitativo. Psico, 23(1), 59-70. doi.org/10.1590/1413-82712018230106

Sit, D. K., \& Wisner, K. L. (2009). The identification of postpartum depression. Clinical obstetrics and gynecology, 52(3), 456-468. doi: 10.1097/GRF.0b013e3181b5a57c

Soares, P. P. N., Ferreira, P. C., dos Santos Calheiros, D., \& Neto, J. L. C. (2016). Saúde mental materna e o desenvolvimento motor de crianças e adolescentes com síndrome de Down. Revista Da Sobama, 17(1), 31-36.

Souza, B. M. S, Souza, S. F., Rodrigues, R. T. S. (2013) O puerpério e a mulher contemporânea: uma investigação sobre a vivência e os impactos da perda de autonomia. Revista da SBPH, RJ, 16(1), 166-184.

Stedile, A., Da Silva, L., \& Hartmann, F. (2013). O desenvolvimento do vínculo Mãe Bebê após o diagnóstico de Síndrome de Down. Revista Saúde Mental em Foco do Cesuca, 2(1), 1-14. https://doi.org/10.5935/ cadernosdisturbios.v17n2p43-55

Tekinarslan, I. C. (2013). A comparison study of depression and quality of life in Turkish mothers of children with Down syndrome, cerebral palsy, and autism spectrum disorder. Psychological Reports, 112(1), 266-287. doi.org/10.2466/21.02.15.PR0.112.1.266-287 
Van Riper, M., Ryff, C., \& Pridham, K. (1992). Parental and family well-being in families of children with down syndrome: A comparative study. Research in nursing \& health, 15(3), 227-235. doi.org/10.1002/nur.4770150309 Vital, A. A. F., Miccas, C., Duarte, C. P., \& D’Antino, M. E. F. (2016). Avaliação de alunos com síndrome de Down: aspectos cognitivo-linguísticos, educacionais e funcionais. Psicologia: Teoria e Prática, 17(3), 177-188. doi.org/10.15348/1980-6906/psicologia.v17n3p177-188

Endereço para correspondência: Tahena Silva Ferreira (Rua Dr. Aristeu dos Santos Ribas, 247, Londrina, PR, 86079-440; (43) 33254546 / (43) 999934547; tahena@gmail.com).

Recebido em: 27/12/2018.

Aprovado em: 17/5/2019.

Publicado em: xx/xx/2019.

\section{Tahena Silva Ferreira}

E-mail:tahena@gmail.com

Titulação Acadêmica: Mestra pelo Programa de Pós-Graduação em Psicologia do Desenvolvimento e Aprendizagem

Afiliação Institucional: Universidade Estadual Paulista “Júlio, de Mesquita Filho", UNESP/Bauru - Brasil.

\section{Bárbara Camila de Campos}

E-mail: badecampos@gmail.com

Titulação Acadêmica: Mestra pelo Programa de Pós-Graduação em Psicologia do Desenvolvimento e Aprendizagem

Afiliação Institucional: Universidade Estadual Paulista “Júlio, de Mesquita Filho", UNESP/Bauru - Brasil.

\section{Olga Maria Piazentin Rolim Rodrigues}

E-mail: olgarolim29@gmail.com

Titulação Acadêmica: $\operatorname{Prof}^{a} \operatorname{Dr}^{\mathrm{a}}$ Adjunta do Programa de Pós-graduação em Psicologia do Desenvolvimento e Aprendizagem

Afiliação Institucional: Universidade Estadual Paulista "Júlio, de Mesquita Filho", UNESP/Bauru - Brasil 